EFFECT OF THE NEPHROTIC SYNDROME ON THE CONCENTRATION OF SERUM COMPLEMENT COMPONENTS. C. Frederic Strife, Elizabeth C. Jackson, Judith Forristal and Clark D. West. Division of Nephrology, Children's Hospital Research Foundation and the Department of Pediatrics, University of Cincinnati, Cincinnati, Ohio 45229.

The concentration of twelve component and four control proteins of the complement system were measured in serum from 43 children with a nephrotic syndrome which subsequently proved to be steroid responsive and from 13 children with focal glomerulosclerosis (FGS) and compared to values from 197 normal subjects. Of classical pathway complement components, $40 \%$ of patients had low $\mathrm{Cla}$ and $20 \%$, low $\mathrm{C} 2$ leve1s. Me an serum levels patients $\mathrm{C}$, $\mathrm{Cl}$ INH and $\mathrm{C} 4 \mathrm{~b}$, were elevated way components, factors $B$ and I were low in one-third while way components, factors $\mathrm{H}$ were commonly elevated. Of the terminal components, only $C 8$ and $C 9$ were 10 . In five FGS patients with hypoalbuminemia without edema, all component levels were normal. With the exception of $\mathrm{Clq}, \mathrm{Cls}$ and $\mathrm{C} 8$, high molecular weight components were in high concentration and low molecular weight components in low concentration. The three exceptions may be explained by the sub-unit structure of $\mathrm{Cl}$ and $\mathrm{C} 8$. From a practical standpoint, the study indicates that edematous a practical standpoint, serum levels of $\mathrm{Clq}$ and $\mathrm{C} 2$, simulating classical pathway complement activation such as commonly occurs in glomerulonephritis. However, low levels of $\mathrm{C} 4$ and possibly $\mathrm{Cls}$ can be used as indicators of classical pathway activation since their levels are not reduced by a nephrotic syndrome.

PROSTACYCLIN DEFICIENCY DOES NOT PLAY A PIVOTAL ROLE 1640 IN HEMOLYTIC UREMIC SYNDROME (HUS). Marie J. Stuart, Roger E. Spitzer, Ronald W. Walenga, Sherry Boone. SunY, Upstate Medical Center, Dept. of Pediatrics, Syracuse, N.Y. A decrease in the prostacyclin (PGI2) stimulating activity of plasma has been reported in children with HUS. Although no study has attempted to assess plasma PGI2 levels themselves, the presence of a decrease in the PGI2 stimulating activity has been equated with a deficiency of PGI2 in HUS. We have conducted an evaluation of plasma prostanoids (PGI2 and TXB2) in 5 children admitted to SUNY during an epidemic of HUS in the northeast and in 23 controls. We were unable to document a pivotal role for PGI2 deficiency in HUS. Using an RIA for plasma 6KPGF bul the stable hydrolysis product of PGI2), patients with HUS demonstrated elevated $6 \mathrm{KPGF} 1 \alpha$ levels of $1.05+0.3$ (1SD) pmol $/ \mathrm{ml}$ when com pared to controls $(0.41 \pm 0.13 ; p<0.0 \overline{0} 1)$. Increased production of PGI2 in HUS was accompanied by a concomitant increase in plasma thromboxane. Compared to a plasma TXB2 level of $0.10+0.06$ in controls, the mean level in patients with HUS was $0.33+\overline{0} .09 \mathrm{pmol} / \mathrm{ml}$ $(p<0.001)$. When the ratio of plasma TXB2 to 6 KPGF $1 \alpha$ was evaluated as an index of proaggregatory and vasoconstrictor versus antiaggregatory and vasodilator influences, no difference was observed between controls $(0.33+0.06)$ and HUS $(0.33+0.08)$. Changes in prostanoid production in HUS appear to reflect vascular injury ( $\uparrow 6 \mathrm{KPGF} 1 \alpha$ and concomitant platelet consumption ( $\uparrow$ TXB2). A deficiency of PGI2 in HUS was an attractive hypothesis that explained the systemic hypertension and the observed thrombocytopenia. Our finding however does not support a role for prostacyclin deficiency, and underscores the heterogenousness of HUS. 1641 PERCUTANEOUS RENAL BIOPSY (PRB) IN INFANTS. Me1anie 1641 Sweet, Ben H. Brouhard, Alok Kalia, Luther B. Travis. Pediatrics, Galveston, Texas.

Percutaneous renal biopsy is a valuable tool in the evaluation of patients with renal disease. There has been hesitation about performing this procedure in very young infants because the greater mobility of the kidneys may increase the risk of complications and result in an inadequate specimen.

Over the past 4 years, we have performed PRB using solely anatomic 1andmarks in 7 infants under 3 months of age. Reason for biopsy, age, clinical features and pathologic diagnosis are listed below.

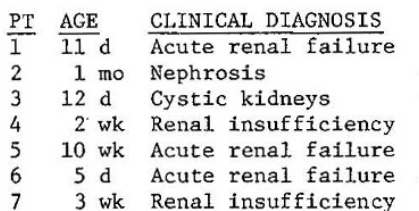

\section{PATHOLOGIC DIAGNOSIS}

\section{Cortical necrosis} Congenital nephrotic syndrome Cortical necrosis

Interstitial nephritis

Cortical necrosis

Acute tubular necrosis

Complications consisted of increases in microscopic hematuria or transient gross hematuria requiring no intervention. All tissue contained $\geqslant 8$ glomeruli and was sufficient for diagnosis.

Blind PRB can be safely performed in small newborns and poses a lesser risk than open biopsy, with adequate specimens being obtained.
1642 LONGITUDINAL STUDY OF RENAL FUNCTION AND MORPHOLOGY IN UNI-NEPHRECTOMIZED RATS. Amir Tejani, Ina Lancman*, (Introduced by Laurence Finberg). Downstate Medical Center, Brooklyn, New York.

To evaluate the long term effects of a $50 \%$ reduction in nephron population, we studied male Sprague-Dawley rats weight (BWT) $250 \pm 20 \mathrm{gm}$. Animals were subjected to either a sham operation (S) or right nephrectomy (N). Animals were maintaine on standard lab chow, Intra-arterial blood pressure (B.P.), glomerular filteration rate (GFR) and renal plasma flow (RPF) were measured at 4,8 and 12 months (Mo.). Animals were then sacrificed, left kidney (LK) weighed and

Mo. LK wt Mean \pm s.

GFR RPF BP Number 0

mmHg Animals

$1.5 \pm 0.43 .1 \pm 0.9 \quad 160 \pm 05.6 *$

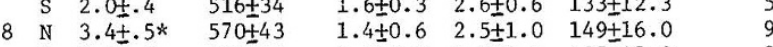

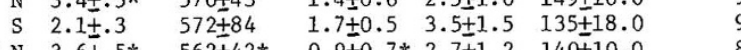

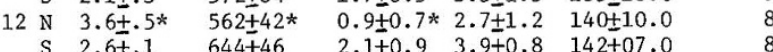

No morphologic changes were noted in 4 and $8 \mathrm{mo}$. S and $N$ animals. Twelve mo. $S$ rats showed $0-2 \%$, segmental glomerulosclerosis. Twelve mo. N rats showed Mean $13 \%$, segmenta1. glomerulosclerosis. Our study shows that in the male rat, a $50 \%$ reduction in renal mass leads to functional and morphological damage to the contralateral kidney over a one year period.

LONG-TERM OUTCOME OF DIFTUSE PROLT

1643 NEPHRITIS. A. Tejani, K. Phadke, R. Khawar, S. Fikr H. Trachtman, Downstate Med. Ctr., SUNY, Brooklyn. We reviewed our experience with diffuse proliferative Lupus Nephritis (D.P.L.N.) in 22 children. Aggressive steroid and ant metabolite therapy prevented end stage renal disease (E.S.R.D.) in 10 patients, but 2 patients died of sepsis. 12 patients have reached E.S.R.D. in a mean of 30 months from diagnosis. Dialysis: 12 patients received hemodialysis from 3 to 23 months (mean 13 mos.). No mortality was recorded while on dialysis but a high rate of morbidity was noted. The 12 Lupus patients accounted for $60 \%$ of all hospitalizations compared to 51 non Lupus children on dialysis who accounted for $40 \%$ of hospitalization (p<.001). One child was able to discontinue dialysis after a period of 9 months.

Transplant: 9 children received 12 transplants (1 patient had 4 transplants). One patient died of sepsis during the 2nd month after transplantation. Graft survival rates at 1 and 4 years were 80 and $65 \%$ respectively. Patients with a successful transplant spent a mean of 25 days in hospital post transplant compared to a mean of 112 days in hospital during dialysis ( $<.05)$. One patient had clinical and histological recurrence within 6 months after a live related transplant. Our study concludes: 1) majority of D.P.L.N. patients will reach E.S.R.D. in 3 years; 2) mortality is due to therapy associated sepsis; 3) dialysis is safe but has a high morbidity and 4 ) successful transplant has the best quality of life but the risk of recurrence in live related donors is a concern.

$\bullet 1644$ CYCLOSPORINE (CY) AS A PRIMARY IMMUNOSUPPRESSIVE, AS A SUBSTITUTE FOR AZOTHIOPRIN (A) AND AS THE SOLE MAINTENANCE DRUG IN RENAL TRANSPLANTS. A. Tejani, H. Trachtman, K. Phadke, M. Khawar and K.M.H. Butt, (Introduced by L. Finberg), SUNY, Downstate Medical Center, Brooklyn, N.Y. We utilized the steroid sparing effect of cyclosporine in 32 Pediatric renal transplants. Nineteen children (Group A) Pediatric renal transplants. Nineteen children (Group A) transplant. In 13 children (Group B), with a stable renal trans plant on conventional therapy (A\&P), CY was substituted for A. In both Groups, we attempted to taper $\mathrm{P}$ with complete withdrawa in 16-20 weeks. Results: Group $A$ had 11 first and 8 2nd or $3 \mathrm{rd}$ transplants. There were 11 cadaveric (C) and 8 live related (L) transplants. Actuarial graft survival at 16 months is $88 \%$. Group $\mathrm{B}$ had $4 \mathrm{C}$ and $9 \mathrm{~L}$ grafts. Mean serum creatinine (M.S.C.) at time of substitution was $1.6 \mathrm{mg} / \mathrm{dl}$. No graft loss has occurred in Group B after substitution. A total of 13 out of 32 children were able to discontinue P. Four of these are receiving $P$ again because of rejection. Nine children have been off $P$ for a mean of $5 \frac{1}{2}$ months (2-14 months), their M.S.C. is $1.06 \mathrm{mg} / \mathrm{d} 1$ (.07-1.5 mg/d1) A.C.T.H. stimulation after discontinuation of $P$ revealed 2 fold rise in serum cortisol from base line value in 12 of 13 children. Growth hormone level measured after methyldopa stimulation showed normal $(>10 \mathrm{ng} / \mathrm{ml})$ response in 5 of 7 children off $P$. We conclude 1) selected C\&L graft patients can discontinue $P$ thus enhancing the growth potential; 2) such children are not at risk of developing Addisonian Crisis and 3) donor and recipient haplotype match or the number of transplants were not of predictive value in $P$ withdrawal. 